\section{Actitud de odontólogos acerca de la atención de pacientes con necesidades especiales}

\section{Attitude of dentists about dental care of patients with special needs}

\section{Resumen}

Objetivo: Evaluar la actitud de los cirujanos dentistas acerca de la atención odontológica de pacientes con necesidades especiales (PNE). Métodos: Estudio transversal que utilizó el método cuantitativo a través de un cuestionario que fue contestado por 60 cirujanos dentistas (CDs) asistentes a una Jornada Odontológica del estado de Río Grande do Sul, Brasil. Resultados: El 73\% de los CDs no atienden este tipo de pacientes, de los cuales: 62,1\% por no saber atenderlos; $91,4 \%$ por no haber recibido un Curso de Pacientes Especiales durante su formación académica; $25,9 \%$ porque requiere mucho tiempo y paciencia y $69 \%$ por no poseer las condiciones emocionales. Por otro lado, el $4 \%$ de los encuestados respondieron si atenderlos; bajo alguna forma de contención, sedación o de ser el caso referirlos a un especialista. El 23\% restante de CDs indicó prestar atención solamente si eran pacientes colaboradores. Conclusiones: La odontología para pacientes con necesidades especiales en el estado de Rio Grande do Sul-Brasil parece no cubrir los requerimientos de estos pacientes, no solo por las dificultades inherentes a los servicios y las especificidades de dichos pacientes, sino debido a los impedimentos relacionados con los propios dentistas, problemas con las técnicas odontológicas y por toda la complejidad que envuelve la atención a personas con deficiencia.

Palabras clave: Atención odontológica; Personas con deficiencia; Servicios de salud.

\section{Artículo Original}

Márcia Cançado Figueiredo ${ }^{1, a, f}$, Andressa Nicoli Haas ${ }^{1, b}$, Débora Scariot 2,a,c,

Geisa Luchsinger Lima ${ }^{1, a, d}$, Ximena Concha Melgar ${ }^{3,1, a, e}$

${ }^{1}$ Universidade Federal do Rio Grande do Sul. Faculdade de Odontologia. Porto Alegre, Rio Grande do Sul, Brasil.

${ }^{2}$ Comissão de Odontologia para Pacientes Especiais do Consejo Regional de Odontologia de Rio Grande do Sul. Porto Alegre, Rio Grande do Sul, Brasil.

${ }^{3}$ Universidad Científica del Sur. Carrera de Estomatología. Lima, Perú.

a Cirujano dentista.

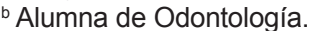

c Especialista en Odontopediatría.

d Especialista en Pacientes con necesidades especiales.

e Magíster en Odontopediatría.

f Doctor en Odontopediatría.

Correspondencia:

Ximena Concha Melgar

Correo electrónico: xconchame@cientifica.edu.pe Calle Gamma C3 urb. Juan XXIII San Borja. Lima-Perú

\section{Coautores: \\ Márcia Cançado Figueiredo \\ mcf195@gmail.com \\ Andressa Nicoli Haas andressahaas@hotmail.com Débora Scariot \\ scariotdebora@hotmail.com \\ Geisa Luchsinger Lima \\ geisalima.rs@gmail.com}

Conflicto de intereses: Los autores declaran no tener conflictos de interés.

Fuente de financiamiento: Autofinanciado.

Fecha de recepción: 26/08/18

Fecha de aceptación: 17/12/18 


\begin{abstract}
Objective: Evaluate the attitude of dental surgeons (DS) in relation to dental care for patients with special needs (PSN). Methods: Transversal study that used the quantitative method through a questionnaire which was answered by 60 DS attending at Dental Conference in the state of Rio Grande do Sul, Brazil. Results: $73 \%$ of the DS do not attend this type of patients, among these: $62.1 \%$ because they do not know how to treat them; $91.4 \%$ because they did not have a Special Patients Course during their academic education; $25.9 \%$ because it requires a lot of time and patience and $69 \%$ because it needs certain emotional conditions. On the other hand, $4 \%$ of the respondents answered that they atended PSN; under some form of containment, sedation or if it was the case, refer them to a specialist. The remaining $23 \%$ of DS indicated paying attention only if they were collaborating patients. Conclusions: Dentistry for PSN in the state of Rio Grande do Sul-Brazil does not seem to satisfy the requirements of these patients, not only due to the difficulties inherent to the services and the specificities of these patients, but also of the impediments related with the dentists themselves, problems with dental techniques and all the complexity involved in caring for people with disabilities.
\end{abstract}

Keywords: Dental care; Disabled persons; Health services.

\section{Introducción}

Personas con deficiencia son aquellas que poseen impedimentos de largo plazo de naturaleza física, mental, intelectual o sensorial; los cuales, en interacción con diversas barreras, pueden obstruir su participación plena y efectiva en la sociedad en igualdad de condiciones con las demás personas. Las personas con deficiencia tienden a presentar mayor riesgo de enfermedades bucales por el uso sistémico de medicamentos, la dificultad en la realización del control de placa bacteriana y hábitos alimenticios precarios. Por lo tanto, dichos pacientes deben recibir atención precoz y cuidados para evitar problemas futuros ${ }^{1,2}$. Además, las personas con necesidades especiales suelen sufrir más problemas dentarios, ausencia de dientes y mayor dificultad para recibir tratamiento odontológico que otros miembros de la población debido a la falta de capacitación del profesional para esta especialidad $^{3}$.

Este comportamiento viene siendo modificado por profesionales que buscan integrar a dichas personas a la sociedad y proporcionar una atención odontológica diferenciada para ellos, de acuerdo con las necesidades de cada uno e incluyéndolos en los sistemas públicos de atención a la salud ${ }^{4}$. Por otro lado, según Silva ${ }^{5}$, la presencia de pacientes con requerimientos especiales en el consultorio privado exige adecuaciones ergonómicas para sus limitaciones, además de la capacitación del cirujano dentista.

Las Directrices Curriculares Nacionales para los Cursos de Odontología en Brasil, en 2002, determinan que la formación del cirujano-dentista debe permitirle actuar en todos los niveles de atención a la salud; para ello, debe recibir una formación generalista ${ }^{6}$. Concluyendo que, el profesional de odontología requiere tener conocimiento para atender todas las necesidades de sus pacientes, independientemente de sus características.

Desde la promulgación de la Ley 9.394/96, las instituciones de educación superior obtuvieron autonomía para la construcción de la malla curricular, posibilitando la inclusión o no de cursos ${ }^{7}$. La Atención de Pacientes Especiales es un curso que se ciñe a la voluntad facultativa de la institución de contar con él. Investigaciones afirman que cuando el futuro profesional de odontología tiene la oportunidad de vivenciar experiencias con pacientes con necesidades especiales durante la carrera, se muestra más seguro y dispuesto a atender a dicha población cuando ya es profesional ${ }^{8,9}$.

Muchas personas con necesidades especiales tienen dificultad de encontrar dentistas dispuestos a cubrir sus requerimientos y que cuenten con servicios accesibles para ellos ${ }^{10,11}$. Muchos cirujanos dentistas rechazan atender pacientes especiales por falta de entrenamiento, preparación, experiencia, entendimiento, miedo y una inhabilidad de establecer vínculo ${ }^{12}$.

El mayor desafío para este tipo de atención es que no envuelve únicamente a la persona con deficiencia, sino también a su familia. Lo cual es medida fundamental para una atención humana, amplia y eficiente, con apoyo psicosocial y orientaciones para la realización de las actividades de vida cotidiana ${ }^{13}$.

En la odontología, hay necesidad de entablar una relación de confianza entre el profesional y sus pacientes, en el sentido de minimizar situaciones amenazadoras y estresantes. El conocimiento científico y habilidad técnica no son suficientes en esta relación, pues cada paciente es único y especial, por exhibir grados diferentes de comportamiento de salud y diferentes dificultades para adecuarse a ciertas situaciones ${ }^{14,15}$.

Dentro del contexto de promoción de la salud bucal, la atención de pacientes con necesidades especiales (PNE) exige conocimiento no siempre disponible en cursos formadores, tanto en pregrado como posgrado. Estas necesidades odontológicas son innumerables, agravadas por la negligencia con las que son abordadas por todo 
el universo de personas que las rodean, sean familiares o hasta profesionales de salud. El área de salud más descuidada tal vez sea la misma odontología. En parte, debido a que la familia se envuelve más con el problema médico, considerado prioritario, relegando la salud bucal para un segundo plano. Otro problema es la falta de cirujanos dentistas con conocimiento suficiente para la atención de estos pacientes ${ }^{16}$.

De acuerdo con la Organización Mundial de la Salud (OMS), 10\% de la población mundial presenta algún desvió de la normalidad ${ }^{17}$. En Brasil, según el Instituto Brasileiro de Geografía y Estadística (IBGE), el porcentaje se eleva a $15 \%$ de PNE ${ }^{18}$, y apenas $3 \%$ recibe atención odontológica ${ }^{1}$. Dado esto, la especialidad de odontología para PNE fue aprobada en el 2001, durante la $2^{\text {a }}$ Asamblea Nacional de Especialidades Odontológicas (ANEO), con el objetivo de diagnosticar, prevenir, tratar y controlar los problemas de salud bucal de estos pacientes. Los cuales presentan un sistema biológico, psicológico y/o social complejo, en percepción y actuación, dentro de una estructura transdisciplinar con otros profesionales de salud y áreas relacionadas con el paciente ${ }^{19}$.

Además, el 28 de abril del 2002 fue publicada la resolución 25/2002 en el Diário Oficial da União - D.O.U. por el Consejo Federal de Odontología (CFO), que reglamentó la especialidad de Odontología para Pacientes con Necesidades Especiales. Actualmente, en el año 2017, están inscritos apenas 30 cirujanos dentistas en dicha especialidad en el Consejo Regional de Odontología del estado de Rio Grande do Sul (CRO/RS) y el acceso a la rehabilitación bucal especializada en la red pública de este estado está restricto a tres Centros de Especialidades Odontológicas (CEO) registrados a la Red de Cuidados a Pacientes con Deficiencia del Ministerio de Salud a través del Programa Brasil Sonriente ${ }^{20}$.

Por lo antes expuesto, este estudio tuvo como objetivo evaluar la actitud de cirujanos dentistas registrados en el Consejo Regional de Odontología del estado de Rio Grande do Sul (CRO/RS) acerca de la atención odontológica a PNE

\section{Métodos}

Estudio transversal que utilizó el método cuantitativo ${ }^{21}$ a través de un cuestionario con preguntas cerradas adaptado de Lawrence et al. ${ }^{22}$. Las variables evaluadas fueron: sexo, edad, años de egresado, si posee especialidad, si se dedica a la docencia, si atiende a PNE y cómo los atiende, motivo por el cual no atiende PNE, si cree que la red básica de salud del estado cubre la atención a PNE y si ejerce sus actividades profesionales en la capital o interior. Dos estudiantes de odontología de la Universidad Federal de Rio Grande do Sul (UFRGS) fueron capacitados para la aplicación del referido cuestionario.
Este fue respondido por $60 \mathrm{CDs}$, quienes firmaron un consentimiento y aceptaron participar por libre y espontánea voluntad, de los 150 reunidos en una Jornada Odontológica llevada a cabo en el Sur de Brasil. Dichos CDs ejercían la profesión en diferentes ciudades del estado de Rio Grande do Sul.

Los datos recolectados fueron almacenados en el programa Microsoft Excel 2013 para ser analizados posteriormente, con tabulación en porcentaje y presentados en frecuencia relativa absoluta.

La consistencia interna del cuestionario fue evaluada a través de la prueba de alfa de Cronbach mediante el mismo programa. Dicha prueba mide las correlaciones entre los ítems que son parte de un instrumento por medio del análisis del perfil de las respuestas ${ }^{23} \mathrm{y}$ posee valores que varían de 0 a 1 , siendo que valores por encima de 0,7 indican buena confiabilidad y relación entre las preguntas. ${ }^{24} \mathrm{El}$ valor obtenido en el presente estudio fue de 0,75 .

El proyecto de esta investigación fue aprobado por el Comité de Ética en investigación de la UFRGS bajo el número CAE 61519316.0.0000.5347.

\section{Resultados}

Conforme con la Figura, el 40,7\% de los CDs participantes eran clínicos generales y el 59,3\% restante contaba con alguna especialidad.

Apenas 3,4\% de los participantes eran docentes. La edad de los encuestados varió de 28,6 a 68,9 años; siendo $57 \%$ de sexo femenino y $43 \%$ de sexo masculino (Tabla).

Se preguntó a los CDs si atendían pacientes con necesidades especiales y el $73 \%$ no lo hacía en ninguna situación. De ellos, el 62,1\% indicó que el motivo era porque no sabía cómo atender dichos pacientes; el $91,4 \%$ porque no recibieron un curso de atención odontológica a pacientes especiales durante su formación académica; el 69\% asumió que no contaba con las condiciones emocionales para tal y el 25,9\% manifestó que no lo realizaba por requerir mucho tiempo y paciencia (Tabla).

El 23\% de los CDs respondió que solo atendía PNE si eran colaboradores y el $4 \%$ restante afirmó que sí atendía dichos pacientes sin discriminación, utilizando algún tipo de contención y/o sedación con autorización del médico responsable del paciente cuando era necesario. De estos, el $70 \%$ informó que trabajaba en la capital (Tabla).

Otra pregunta realizada a los entrevistados fue: ¿Ud. presta servicio odontológico en alguna institución que atienda personas con deficiencias múltiples?, dando como resultado $88,3 \%$, de respuestas negativas. Y en cuanto a su opinión sobre la situación de dichos pacientes en la red básica de salud del estado (Sistema Único de Salud - SUS), el 98,3\% reveló que no se cubre la atención odontológica a este segmento (Tabla). 
Tabla. Variables evaluadas en el cuestionario de atención a PNE

\begin{tabular}{|c|c|c|c|}
\hline Variable & & $\mathbf{n}$ & $\%$ \\
\hline \multirow{4}{*}{ Edad } & 20 a 30 años & 20 & 33 \\
\hline & 31 a 40 años & 20 & 33 \\
\hline & 41 a 50 años & 13 & 22 \\
\hline & 51 a más & 7 & 12 \\
\hline \multirow{2}{*}{ Sexo } & Femenino & 34 & 57 \\
\hline & Masculino & 26 & 43 \\
\hline \multirow{3}{*}{ Realiza atención a PNE } & No & 44 & 73 \\
\hline & Solamente pacientes colaboradores & 14 & 23 \\
\hline & $\mathrm{Si}$ & 2 & 4 \\
\hline \multirow{4}{*}{ Motivo por el cuál no atiende PNE } & No sé cómo atenderlos & 36 & 62,1 \\
\hline & $\begin{array}{l}\text { No recibí un curso de atención odontológica a PNE durante } \\
\text { mi formación académica }\end{array}$ & 53 & 91,4 \\
\hline & No tengo condiciones emocionales & 40 & 69 \\
\hline & Requiere mucho tiempo y paciencia & 15 & 25,9 \\
\hline \multirow{3}{*}{$\begin{array}{l}\text { Presta servicio odontológico en alguna institución } \\
\text { que atienda PNE }\end{array}$} & No & 53 & 88,3 \\
\hline & $\mathrm{Si}$ & 7 & 11,7 \\
\hline & No sabe & 0 & 0 \\
\hline \multirow{3}{*}{$\begin{array}{l}\text { Cree que la red básica de salud cubre la atención a } \\
\text { PNE }\end{array}$} & No & 59 & 98,3 \\
\hline & $\mathrm{Si}$ & 1 & 1,7 \\
\hline & No sabe & 0 & 0 \\
\hline \multirow{2}{*}{ Si atiende PNE, ¿En qué región trabaja? } & Capital (Porto Alegre) & 42 & 70 \\
\hline & Interior de Rio Grande do Sul & 18 & 30 \\
\hline
\end{tabular}

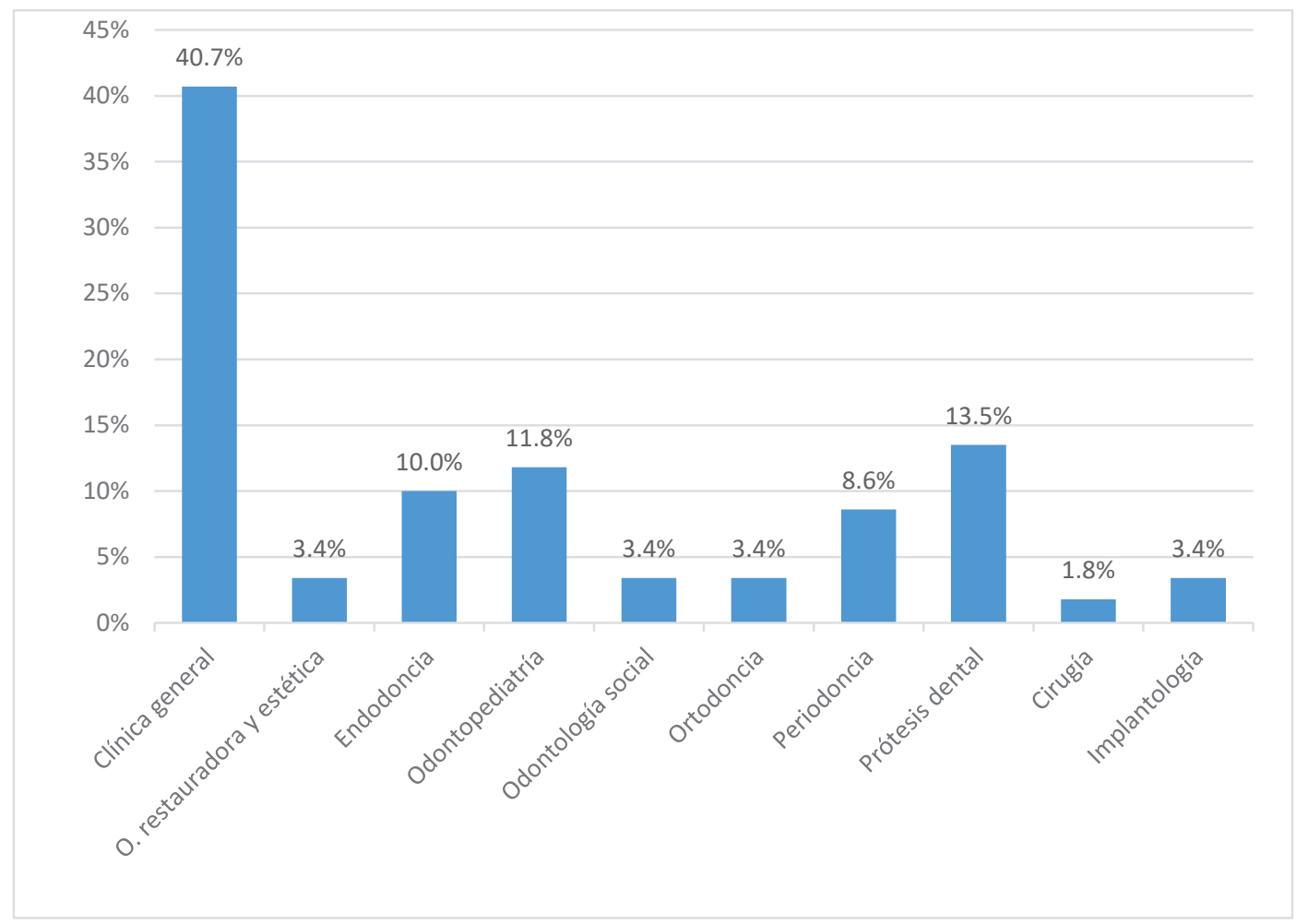

Figura. Distribución de especialidades de los participantes 


\section{Discusión}

La resolución 25/2002, publicada en el Diário Oficial da União - D.O.U. - el 28/05/2002 por el Consejo Federal de Odontología, reglamentó la especialidad de Atención a Pacientes con Necesidades Especiales con la intención de capacitar a los CDs para la atención de personas que necesitan cuidados odontológicos especiales durante toda su vida o por un período ${ }^{20}$. Actualmente, apenas 30 CDs se encuentran registrados en Rio Grande do Sul en dicha especialidad. Ella exige del CD el conocimiento de la complejidad orgánica e identificar la deficiencia y los disturbios que envuelven el comportamiento y la personalidad de estos pacientes. Es una especialidad que permite al CD especialista adquirir una "sensibilidad social”. Además, debe reconocer las infecciones bucales, determinar el tipo de lesión o agente etiológico y otros datos relevantes para el diagnóstico y tratamiento de dichos pacientes ${ }^{5}$.

Según los resultados de este estudio; el principal motivo por el que los CDs no atendían pacientes con necesidades especiales era el no haber recibido dicha preparación durante su formación académica. Esto también fue observado por Figueiredo ${ }^{8}$ en su tesis de maestría, quien visitó las Facultades de Odontología de la región metropolitana de São Paulo y solicitó información sobre la inserción de disciplinas de atención a pacientes especiales en la formación de los profesionales a nivel de pregrado y posgrado. También investigó la formación profesional y técnica del coordinador del curso; la duración del mismo (semestral o anual) y su forma de presentación (teórico o teórico práctico); número de docentes, alumnos y su proporción; localización del curso en la malla curricular; contenido programado; si estaba vinculado a otro curso; número de pacientes atendidos y protocolo de referencias. Su investigación mostró que la incorporación de Atención a Pacientes especiales en la formación de los futuros dentistas aún era insuficiente e incompleta. Observaciones análogas fueron registradas y los principales motivos mencionados fueron la falta de espacio en la malla curricular, de motivación de los dirigentes de las instituciones, de preparación de los profesores e ineptitud de los alumnos que comenzaban sus actividades prácticas.

Casamassimo ${ }^{25}$, en el 2004, afirmó que los profesionales egresados presentaban poca disposición en atender pacientes especiales por el poco tiempo destinado a su aprendizaje durante el pregrado. Sus investigaciones mostraron que los CDs generales desearían haber recibido más entrenamiento para este tipo de atención, lo cual impactaría directamente en su comportamiento y actitudes con respecto a ello.

Otro aspecto interesante encontrado en el mismo estudio, y que concuerda con Fonseca et al. ${ }^{26}$, es que el hecho de que la odontología aun no cubra todas las necesidades de los pacientes especiales puede no estar directamente relacionado con las dificultades inherentes a los servicios o hasta con las necesidades de dichos pacientes. Mas puede estar relacionado a los propios CDs; no a las dificultades en las técnicas odontológicas, sino a toda la complejidad que exige este tipo de atención. Estas dificultades pueden estar relacionadas a aspectos humanos, de orden moral, filosófica y psicológica, y también a no conseguir cubrir las necesidades de los pacientes. Para los mismos autores ${ }^{26}$ es realmente necesario cambiar la "visión" en todos los sentidos y reconocer las necesidades de los servicios públicos de salud disponibles para que sean efectivos y permitan que las acciones de salud sean inclusivas con las personas con deficiencias. La odontología puede contribuir de modo relevante para la vida de los pacientes especiales y el contacto con dichos pacientes desde pregrado puede ser importante para que el futuro CD desenvuelva habilidades técnicas y aspectos humanos fundamentales para su práctica profesional, así como propiciarle experiencias emocionales que lo ayudarán a conquistar actitudes y valores necesarios para un buen desempeño de sus funciones.

Este hecho relatado fue identificado de cierta forma en la presente investigación; por la forma en que clasificaron a los pacientes especiales que eran atendidos; es decir, los "más colaboradores" y/o aquellos que son "más difíciles". Dejando implícita una predilección por atender pacientes "especiales" que se comporten bien, lo cual es ejemplo de cierto descompromiso con el ser humano. Es interesante también destacar que más de la mitad de CDs entrevistados contaban con alguna especialidad registrada en el CRO/RS y, aun así, no atendían pacientes especiales en su consultorio odontológico.

Muchos CDs entrevistados consideraron el encaminar estos pacientes, como si todas las dificultades en atender pacientes especiales dependiesen solamente de un sistema de referencia y contra referencia que fuese efectivo. Este resultado fue semejante al de Lawrence et al. ${ }^{22}$ en el 2014, quienes llevaron a cabo entrevistas basadas en un cuestionario semiestructurado, el cual aplicaron a 44 CDs de una red básica de salud de una capital del nordeste brasilero. El objetivo fue conocer el acceso de los pacientes especiales al servicio de salud bucal en el Sistema de Salud del estado. Se obtuvo como resultados que 36 CDs $(81,8 \%)$ atendían PNE, 29 CDs $(65,9 \%)$ realizaban examen clínico y orientaciones de higiene bucal a PNE y 5 CDs $(11,3 \%)$ realizaban examen clínico a PNE y los referían a un especialista. Solamente nueve de los CDs entrevistados $(20,5 \%)$ tuvieron algún curso relacionado a PNE durante su formación. La evidenció que la red pública presentaba inconvenientes en resolver los problemas bucales, por lo que era necesario encaminar a los pacientes. Los autores concluyeron que es necesario que los profesionales sean sensibilizados en asistir a PNE en su integridad.

Esta dificultad no está limitada a Brasil. Parraguez ${ }^{27}$ relata que en Chile, en el área de odontología, existe poco conocimiento sobre el tratamiento de pacientes en situación de discapacidad. La formación académica no ofrece conocimientos prácticos sobre el tratamiento de dichos pacientes. Además, en los servicios públicos dentales, no existe un tratamiento preferencial para ellos; esto generalmente por escaparse de las metas establecidas por el ministerio de salud. El estado chileno no ha asumido, 
en la totalidad, el rol de dar garantías de integración, rehabilitación y accesibilidad a este segmento de la población; las herramientas entregadas a los servicios públicos son escasas o nulas y, a nivel social, existe poca conciencia e información del problema.

El 2017 en Colombia, Giraldo-Zuluaga et al. ${ }^{28}$ concluyeron que la capacitación para el odontólogo en este campo no es fácil, dado que no se cuenta con suficientes programas que cubran de manera completa la formación del profesional en este ámbito. El propósito de su artículo fue realizar una revisión de tema sobre algunos tipos de discapacidades, las manifestaciones bucales más comunes, el manejo odontológico y estrategias de comunicación alternativa apropiada para brindar una atención de calidad.

Por otro lado, Stiefel ${ }^{29}$ afirmó que la atención en salud bucal para pacientes con deficiencia es un cuidado en salud que recibe poca atención. Él estima que en Estados Unidos, la mitad de la población con deficiencia no consigue encontrar recursos profesionales que brinden informaciones apropiadas y necesarias para el cuidado en salud bucal. La falta de acceso a servicios odontológicos para este creciente segmento de la población está alcanzando niveles críticos y es un dilema nacional.

El 2017, Alamoudi et al. ${ }^{30}$ afirmaron que Arábia Saudita tuvo un rápido desarrollo económico en las últimas décadas; lo que se reflejó en la mejoría de los servicios de salud y en la disminución de la mortalidad, y como consecuencia una mayor probabilidad de sobrevivencia para niños con deficiencia. No obstante, infelizmente, la investigación reveló una triste realidad: los dentistas afirmaron que su educación profesional no los preparó para la atención de PNE y relataron un bajo nivel de autoconfianza para lidiar con dichos casos. En contraste, la mayoría de los entrevistados mostró voluntad en mejorar sus conocimientos y habilidades en el área.

Puesto que la muestra del presente estudio fue muy pequeña, no podemos concluir que la odontología para PNE en el estado de Rio Grande do Sul-Brasil no cubre los requerimientos de estos pacientes. Sin embargo, observamos que existen impedimentos relacionados con los propios dentistas, por problemas con las técnicas odontológicas y manejo de la complejidad que envuelve la atención a personas con deficiencia, que manifiestan presentar por no haber recibido capacitación adecuada al respecto durante su formación profesional. Sugerimos que este estudio debe ser continuado y desarrollado en otros estados y países, para que pueda incentivar investigaciones en el área de odontología de pacientes con necesidades especiales e intercambio de conocimientos.

\section{Referencias bibliográficas}

1. Varellis MLZ. O paciente com necessidades especiais na odontologia - manual prático. São Paulo: Livraria Santos Editora; 2005.

2. Toledo OA. Odontopediatria - Fundamentos para a prática clínica. 3. ed. São Paulo: Editora Premier; 2005.
3. Marra O.S. Dificuldades encontradas pelos responsáveis para manter a saúde bucal em portadores de necessidades especiais [Dissertação de Mestrado]. [Rio de Janeiro]: Escola de Odontologia, Universidade do Grande Rio; 2007.

4. Carvalho ML. Deficiente? Quem? Cirurgiōes-dentistas ou Pacientes com necessidades especiais? Em extensão. 2004;4(1):65-71.

5. Silva ZCM. Avaliação do perfil dos pacientes com necessidades especiais. Revista Odonto Ciência - Fac. Odonto/PUCRS. 2005;20(50):313-18.

6. Ministério da Educação. Resolução $\mathrm{n}^{\circ} \mathrm{CNE} / \mathrm{CES}$ 3/2002 de 19 de fevereiro de 2002. Diretrizes Curriculares Nacionais dos Cursos de Farmácia e Odontologia. Brasília; 2002.

7. Ministério da Educação. Lei no 9.394/96, de 20 de dezembro de 1996. Estabelece as diretrizes e bases da educação nacional. Brasília; 1996.

8. Figueiredo JR. Estratégia para a provisão de cuidados no atendimento odontológico a pacientes portadores de deficiência [dissertação de mestrado]. [São Paulo]: Faculdade de Odontologia, USP; 2002.

9. Oliveira LFA. Oliveira CCC. Gonçalves SRJ. Impacto de um programa de educaçáo e motivaçáo de higiene oral direcionado a crianças portadoras de Necessidades especiais. Odontologia Clín-Científ. 2004;3(3):187-192.

10. Ministério da Saúde. Portaria n. ${ }^{\circ} 199$ de 30 de janeiro de 2014. Política Nacional de Atençáo Integral às Pessoas com Doenças Raras. Diretrizes para Atenção Integral às Pessoas com Doenças Raras no âmbito do Sistema Único de Saúde (SUS). Brasília; 2014.

11. Fassina AP. Análise da disciplina de pacientes portadores de necessidades especiais na Faculdade no Brasil em 2005 [Dissertação de Mestrado]. [São Paulo]: Faculdade de Odontologia, USP; 2006.

12. Lee MM, Sonis AL. An instrument to assess dental Students' attituds toward the handicapped. Spec Care Dentist. 1983;3:117-23.

13. Ministério da Saúde. Política Nacional de Saúde da Pessoa com Deficiência. Brasília; 2010.

14. Bervique JA. Medeiros EPG. O processo de comunicação. In: Bervique JA. Ciências da conduta na área da saúde: um programa modularizado de introdução e aplicação à odontologia, medicina e enfermagem. São Paulo: Panamericana; 1980.

15. Moraes ABA, Pessotti I. A psicologia do cirurgião-dentista. En: Moraes ABA. Pessotti I. Psicologia aplicada à odontologia. São Paulo: Sarvier; 1985.

16. Silva LCP. Cruz RA. Odontologia para Pacientes com Necessidades Especiais: Protocolos para o Atendimento Clínico. São Paulo: Santos editora; 2009.

17. World Health Organization. Relatório Mundial de Saúde. Geneva: WHO; 2010.

18. Instituto Brasileiro de Geografia e Estatística (IBGE). Censo Demográfico de 2010. Brasil; 2010

19. Conselho Federal de Odontologia. Resolução 22/2001 de 27 de dezembro de 2001. Normas sobre anúncio e 
exercício das especialidades odontológicas e sobre cursos de especialização. Brasília; 2001.

20. Conselho Federal de Odontologia. Resolução 25/2002 de 28 de maio de 2002. Estabelece as áreas de competência para atuação dos especialistas em Disfunção Têmporo-Mandibular e Dor Orofacial; Odontogeriatria; Odontologia do Trabalho; Odontologia para Pacientes com Necessidades Especiais e em Ortopedia Funcional dos Maxilares e dá outras providências. Brasilia; 2002

21. Minayo MCS. Pesquisa social: teoria, método e criatividade. Porto Alegre: Vozes; 2002.

22. Lawrence H, Sousa LP, Gonçalves FL, Saintrain MVL, Vieira APGF. Acesso à Saúde Bucal Pública pelo paciente especial: A ótica do Cirurgião-Dentista. Rev Bras. Prom. Saúde. 2014;27(2):190-7. DOI: http://dx.doi. org/10.5020/2620

23. Da Hora H, Monteiro G, Arica J. Confiabilidade em questionários para qualidade: um estudo com o Coeficiente Alfa de Cronbach. Produto \& Produção. 2010;11(2):85-103.

24. Molina M, Aranda M, Flores M, López M. Utilización del alfa de Cronbach para validar la confiabilidad de un instrumento de medición de satisfacción del estudiante en el uso del software Minitab. 11th Latin American and Caribbean Conference for Engineering and Technology 2013; Cancun, Mexico. [Consultado el 18 de abril de 2018]. Accesible en:http://www.laccei.org/LACCEI2013- Cancun/
25. Casamassimo PS, Seale NS, Ruehs K. General dentists' perceptions of educational and treatment issues affecting access to care for children with special health care needs. J Dent Educ. 2004;68:23-8.

26. Fonseca ALA, Azzalis LA, Fonseca FLA, Botazzo C. Análise qualitativa das percepçóes de cirurgióes dentistas envolvidos nos atendimentos de pacientes com necessidades especiais de serviços públicos municipais Rev Bras Cresc. Desenv. Humano. 2010;20(2):208-216.

27. Parraguez CG. Percepción de atención dental de pacientes en situacion de discapacidad atendidos en reclinador de silla de ruedas [trabajo de investigación para optar al título de cirujano-dentista]. [Santiago]: Facultad de Odontología, Universidad de Chile; 2013.

28. Giraldo-Zuluaga MC, Martínez-Delgado CM, Cardona-Gómez N, Gutiérrez-Pineda JL, Giraldo-Moncada KA, Jiménez-Ruíz PM. Manejo de la salud bucal en discapacitados. Artículo de revisión. Rev CES Odont. 2017;30(2):23-36. DOI: http://dx.doi.org/10.21615/ cesodon.30.2.3

29. Stiefel DJ. Dental Care Considerations for Disabled Adults. Spec Care Dentist. 2002;22(3):26-39.

30. Alamoudi NM, Almushayt AS, El Derwi DA, Mirdad LH, El-Ashiry EA. Dentists' Perception of the Care of Patients with Special Health Care Needs in Jeddah, Saudi Arabia. J Oral Hyg Health. 2017;5(3). DOI: 10.4172/2332-0702.1000226 
